\title{
Use of voltammetry for in vitro equilibrium and transport studies of ionisable drugs
}

\author{
Matěj Velický ${ }^{1, *}$, Andrew N.J. Rodgers ${ }^{1}$, Robert A.W. Dryfe ${ }^{1}$ and Kin Tam ${ }^{2}$ \\ ${ }^{1}$ School of Chemistry, University of Manchester, Oxford Road, Manchester M13 9PL, UK \\ ${ }^{2}$ Faculty of Health Science, University of Macau, Macau, China \\ *Corresponding Author: E-mail: matej.velicky@googlemail.com; Tel.: +44-161-306-2770; Fax: +44-161-275-4598
}

Received: November 29, 2013; Published: September 16, 2014

\begin{abstract}
In this review, we will briefly outline the voltammetric investigations of the transfer of ionisable drugs at the interface between two immiscible electrolyte solutions. The voltammetric techniques enable the determination of some key in vitro properties of ionisable drugs, including partition coefficient, diffusion coefficient and membrane permeability. Some successful applications will be highlighted, together with the background methodologies.
\end{abstract}

\section{Keywords}

Cyclic voltammetry; partition coefficient; diffusion coefficient; permeability; ITIES.

\section{Introduction}

The transport properties of a pharmaceutical generally refer to its diffusion coefficient and membrane permeability, whereas the equilibrium state can be described with the use of the partition coefficient. These parameters underpin the physicochemical characteristics of a drug molecule, and provide means to assess the molecule's transport through membranes and/or lipophilic media. For instance, the determination of the partition coefficient yields its lipophilicity, which represents the affinity of a compound for a lipidic environment. The diffusion coefficient, which is a size dependent parameter, determines how quickly the molecules diffuse within either aqueous or organic environments, while membrane permeability can be described by the rate of permeation of the molecule across an artificial membrane. These parameters are useful for estimation of drug disposition events such as passive absorption and modeling the drug distribution among different body compartments. Therefore, the determination of these parameters is of considerable interest in drug discovery and development.

Over $60 \%$ of marketed drugs are ionisable under physiological conditions [1]. The importance of ionisable forms, and their charge, on the overall transport properties of drugs have traditionally been underestimated mainly due to the lack of reliable methods for determination. The introduction of voltammetric techniques has offered new opportunities for evaluation of the transport properties and understanding of the transport processes of ionisable drugs. In this mini review, voltammetric investigations made on the transfer of ionisable drugs at the interface between two immiscible electrolyte solutions (ITIES) will be discussed. In particular, cyclic voltammetry has been used extensively to investigate 
the transfer characteristics of charged species. Particular emphasis will be placed on the determination of partition coefficients, diffusion coefficients and membrane permeability.

\section{Determination of partition coefficients}

The ADMET (absorption, distribution, metabolism, excretion and toxicity) of a drug is generally governed by both its chemical structure and physiochemical properties, such as lipophilicity, solubility and ionisation. Of these physiochemical properties, lipophilicity is widely regarded as the most important parameter to describe the affinity of a drug to a lipophilic phase and is used in both the design of drugs and assessment of their performance [2-4].

The study of drug partition across an interface between immiscible aqueous and organic phases is an established way to characterise the lipophilicity of drugs and the resulting partition coefficient $(P)$ is a common measure of lipophilicity. A partition coefficient is simply the ratio of the activity of a solute in both phases in equilibrium, as expressed in Eq. (1), in which $a_{A}^{\mathrm{w}}$ and $a_{\AA}^{\circ}$ are the activities of solute $\mathrm{A}$ in the aqueous and organic phases, respectively.

$$
P=\frac{a_{\AA}^{\circ}}{a_{\AA}^{w}}
$$

Due to the wide range of their values, partition coefficients are usually quoted on a logarithmic scale, as $\log P$. Shake-flask experiments and potentiometry are traditional methodologies used for determining partition coefficients in the standard water $\mid n$-octanol system [5-9]. However, the partition coefficients of ionised fractions of drugs were usually ignored. The increasing interest in partitioning of fully or partially ionised drugs prompted the search for new methodologies to determine partition coefficients [10-12].

One of the first applications of electrochemistry for the determination of partition coefficients was that of Kontturi and Murtomäki [10], in which cyclic voltammetry at the ITIES was used. Studies at the ITIES are analogous to electrochemistry at the more common solid electrode/solution interface, with the solid electrode being replaced by a second liquid phase. The application of a potential across the ITIES, known as the Galvani potential $(\Delta \varphi)$, produces current as a result of charge transfer across the ITIES. Both liquid phases must be rendered conductive through the dissolution of an aqueous and an organic electrolyte, which remain in their respective phases, in order to apply a potential across the ITIES. Conventionally, the Galvani potential is defined as the potential difference between the aqueous and organic phase, with the sign of the Galvani potential assigned to the aqueous phase, e.g. positive Galvani potential corresponding to the aqueous phase being positively polarised with respect to the organic phase. A diagram of an ITIES setup is shown in Fig. 1. As in conventional solid electrode|solution electrochemistry, the current can arise from electron transfer across ITIES and additionally it can also arise from the transfer of ions across ITIES [13-17]. This renders the ITIES ideally suited to the study of ion partitioning across two immiscible phases, which combined with traditional techniques allows for the contribution of ions and neutral compounds to be determined, as shown in the studies of Bouchard et al. [18,19], Reymond et al. [11], and Nagatani et al. [20]. 


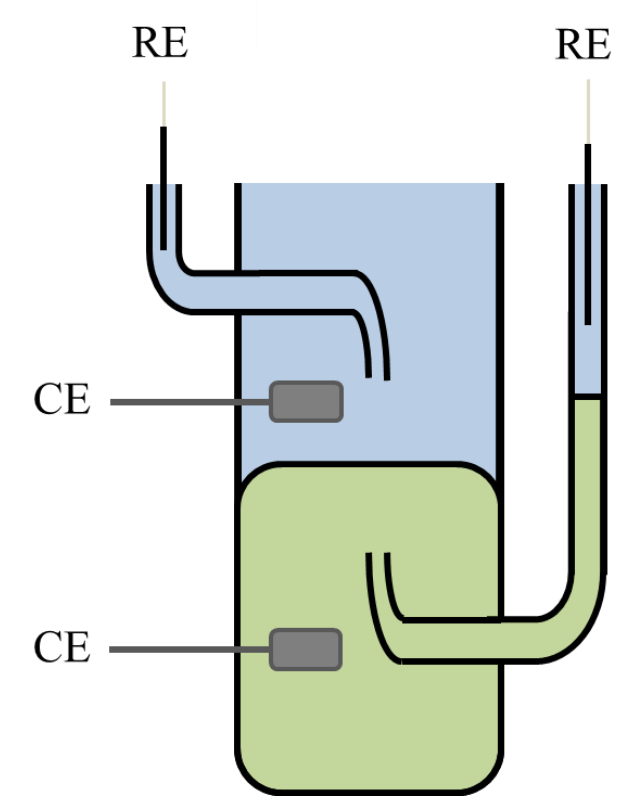

Figure 1. A schematic of the interface between two immiscible electrolyte solutions (ITIES), with an organic (green) and an aqueous (blue) phase, set-up for electrochemical measurement. Each phase contains a counter and reference electrode (CE and RE, respectively), which allow application of potential difference (voltage) between the two liquid phases.

Partition coefficients are simply a measure of solute's affinity for either phase of a liquid |liquid system, as are Gibbs energies of transfer across the ITIES, and therefore the two are directly related. This is demonstrated by Eq.(2), where $P_{\mathrm{i}}$ and $P_{\mathrm{i}}^{0}$ are the partition coefficient and standard partition coefficient, respectively, of ion i, $\Delta \varphi$ is the Galvani potential, $\Delta G_{\mathrm{tr}, \mathrm{i}}^{0, \mathrm{w} \rightarrow 0}$ is the standard Gibbs energy of transfer for ion $\mathrm{i}$ from the aqueous phase to the organic phase, $z_{\mathrm{i}}$ is the charge of ion $\mathrm{i}, F$ is the Faraday constant, $R$ is the universal gas constant and $T$ is the temperature $[14,15]$.

$$
\log P_{\mathrm{i}}=-\frac{\Delta G_{\mathrm{tr}, \mathrm{i}}^{0, \mathrm{w} \rightarrow \mathrm{o}}}{R T \ln 10}+\frac{z_{\mathrm{i}} F}{R T \ln 10} \Delta \varphi=\log P_{\mathrm{i}}^{0}+\frac{z_{\mathrm{i}} F}{R T \ln 10} \Delta \varphi
$$

The Gibbs energy of transfer for an ion is itself related to the standard transfer potential of the ion, which can be determined from the half-wave potential of the peak on a cyclic voltammogram (CV) corresponding to the transfer of the ion from one phase to the other. The standard partition coefficient $\left(P_{i}^{0}\right)$ is the partition coefficient of ion i when no potential is applied across the ITIES, while the partition coefficient $\left(P_{i}\right)$ is the partition coefficient of ion $i$ at a given Galvani potential, as defined by Eq. (2).

As a result of its poor polarisability, $n$-octanol is not a suitable organic solvent for ITIES studies. In the paper of Kontturi and Murtomäki [10], 1,2-dichloroethane (DCE) was chosen as a replacement for $n$ octanol, because of their similar electrical properties, including relative permittivity and dipole moment, and the fact that DCE is sufficiently polarisable to allow the transfer of ions across the water|DCE interface. The water|DCE system has now become the standard for determining partition coefficients of drugs using the ITIES [20-23]. Kontturi and Murtomäki [10] determined partition coefficients at aqueous $\mathrm{pH} \approx 2$, at which the drugs under investigation existed in a protonated form. As mentioned previously, it is not possible to electrochemically determine the partition coefficients of neutral drugs using the ITIES. In order to circumvent this, Kontturi and Murtomäki calculated the $\Delta G_{\mathrm{tr}}^{0, \mathrm{w} \rightarrow 0}$ for $\mathrm{H}^{+}$, converted it to the standard 
transfer potential and then subtracted its contribution from the overall half-wave transfer potential used for calculating the partition coefficient for each drug. Therefore the calculated partition coefficients corresponded to the neutral forms of the drugs.

$n$-Octanol and DCE are not identical solvents and partition coefficients determined using one solvent are not directly comparable with the other. This is problematic, as the water|n-octanol system has long been established as the standard system for the study of drug partitioning and large databases of partition coefficients have already been determined using this system. Initially, Kontturi and Murtomäki plotted $\log P_{\text {oct }}$ values from the literature against their own $\log P_{\text {DCE }}$ values and found two separate trends, both with good linearity, for proton donors and proton acceptors. Bouchard et al. [21] adopted a similar approach, finding separate linear trends for $\mathrm{H}$-bond-donor and non-H-bond-donor drugs, and also introduced the parameter $\Delta \log P_{\text {oct-DCE, }}^{N}$ which is simply the difference between $\log P_{\text {oct }}$ and $\log P_{\text {DCE }}$ for neutral drug molecules. The value of $\triangle \log P^{\mathrm{N}}{ }_{\text {oct-DCE }}$ is positive when a drug is more attracted by $n$-octanol than DCE and vice versa. It was found that $\triangle \log P^{\mathrm{N}}{ }_{\text {oct-DCE }}$ was positive for $\mathrm{H}$-bond-donor compounds and negative for non$\mathrm{H}$-bond-donor compounds, which is explained by both the greater solubility of water in $n$-octanol and the greater H-bonding capacity of $n$-octanol, relative to DCE. Thus $\Delta \log P^{N}{ }_{\text {oct-DCE }}$ can be used to characterise the $\mathrm{H}$-bond-donor capacity of drugs, although care must be taken during analysis, as drugs which form intramolecular $\mathrm{H}$-bonds acted as non-H-bond-donor drugs in this study.

Jing et al.[24] were able to sufficiently polarise a water|n-octanol nano-interface, supported at the tip of a nano-pipette, and determine partition coefficient for the anionic form of lauric acid. However, as the authors themselves acknowledge, the potential window obtained was only $400 \mathrm{mV}$, which is quite restrictive for investigation of a wide range of partition coefficients.

Gulaboski et al.[25] developed an interesting new methodology for studying the water $\mid n$-octanol interface electrochemically. A droplet of $n$-octanol containing an electrochemically active compound, usually decamethylferrocene (DMFc), is attached to a graphite electrode and immersed in an electrolytic aqueous solution, which contains an anion of interest. The DMFc is oxidised to DMFc ${ }^{+}$at the three-phase boundary between water|graphite|octanol and the anion the transfers across the liquid|liquid interface and into the $n$-octanol droplet in order to compensate the charge and maintain electroneutrality, as illustrated in Fig. 2. This method requires that the Gibbs energy of transfer of DMFc ${ }^{+}$from $n$-octanol to water is larger than that of the anion from water to $n$-octanol. Once the standard redox potential of DMFc/DMFc ${ }^{+}$in $n$-octanol (which was calculated using extrapolated standard Gibbs energy of transfer values of various halide anions from other $n$-aliphatic solvents) was known, the potential of the peak on the $\mathrm{CV}$ caused by the reaction was related to the standard transfer potential of the anion and thus the partition coefficient of the anion could be calculated. The methodology of Gulaboski et al. was further tested by Bouchard et al. [26], in a study which looked at 26 different anions. A good linearity was found between partition coefficients determined in their study and those previously determined using the water|DCE system.

When using the DMFc/DMFc ${ }^{+}$redox couple, the three-phase system is only suitable for determining the partition of anions across the water|n-octanol interface. Quentel et al. [27] sought to address this by using lutetium bis(tetra-tert-butylphthalocyaninato) $\left(\mathrm{Lu}\left[t \mathrm{Bu}_{4} \mathrm{Pc}\right]_{2}\right)$, which can be oxidised or reduced to the cation $\mathrm{Lu}\left[t \mathrm{Bu}_{4} \mathrm{PC}\right]_{2}{ }^{+}$or the anion $\mathrm{Lu}\left[t \mathrm{Bu}_{4} \mathrm{PC}\right]_{2}{ }_{2}$, respectively, therefore allowing the study of both anion and cation transfer using the same redox species. Both $\mathrm{Lu}\left[t \mathrm{Bu}_{4} \mathrm{PC}\right]_{2}^{+}$and $\mathrm{Lu}\left[t \mathrm{Bu}_{4} \mathrm{PC}\right]_{2}^{-}$are strongly lipophilic and therefore suited for this method. The $\Delta G_{\mathrm{tr}, \mathrm{i}}^{0, \mathrm{w} \rightarrow n \text {-octanol }}$ was calculated for several alkali metal and organic cations and whilst these cations are not pharmaceutically relevant, the principle of using the three-phase 
system for cation transfer was demonstrated well. The entire methodology is an elegant bridge between the water $\mid n$-octanol system and electrochemical methods for partition coefficient determination.

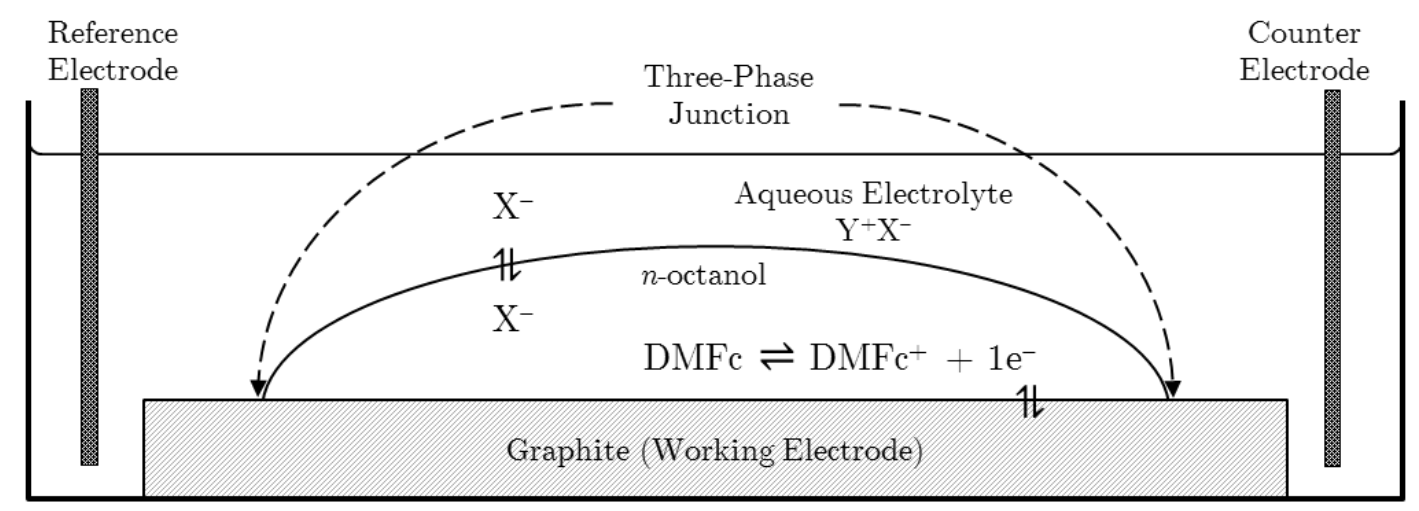

Figure 2. Schematic showing the oxidation of decamethylferrocene (DMFc) in an n-octanol droplet supported on a graphite electrode immersed in an aqueous solution, followed by transfer of an anion $X$ across the liquid/liquid interface in order to maintain electroneutrality.

Whilst the partitioning of neutral compounds is not dependent on either potential or $\mathrm{pH}$, the partitioning of ions is dependent on both. With $\mathrm{pH}$ being an important condition in vivo, it is useful to have an understanding of how both potential and $\mathrm{pH}$ affect the partitioning of ions. Reymond et al. [28] developed ionic partition diagrams for this very purpose. Such diagrams are plots of Galvani potential against $\mathrm{pH}$, similar to the Pourbaix diagrams used in corrosion science, and act as a visual representation of which ions predominate, and in which phase, at a given potential and $\mathrm{pH}$. In addition, they give a description of the mechanisms via which the ions transfer between the two phases, be it simple iontransfer, or a proton-coupled transfer, as either potential or $\mathrm{pH}$ is varied $[19,29]$.

An ionic partition diagram for the zwitterionic drug cetirizine, from the study of Bouchard et al. [19] is shown in Fig. 3, with a more detailed diagram showing mechanisms of phase transfer over a smaller range of potentials and $\mathrm{pH}$, shown in Fig. 4. The theoretical lines in Fig. 3 represent regions in which the concentrations of the species on either side of the line are equal. The horizontal lines are defined by the standard transfer potentials of the species $\mathrm{XH}_{3}{ }^{2+}(w), \mathrm{XH}_{2}{ }^{+}(w)$ and $\mathrm{X}_{(0)}$, where $\mathrm{X}=$ cetirizine. The vertical lines are defined by the three $\mathrm{p} K_{\mathrm{a}}$ values of cetirizine, 2.12, 2.90 and 7.98. The lines of finite gradient represent the transfer of species across the water|DCE interface, which are coupled with a proton exchange, as can be seen from Fig. 3.

lonic-partition diagrams represent the culmination of electrochemical studies of ion-partitioning to this date and provide a valuable first port of call when wishing to understand the partitioning behaviour of an ionised drug under particular conditions of potential and $\mathrm{pH}$. Whilst there is still work to be done in bridging the water|n-octanol and water|DCE systems, the contrasting information provided by these systems has actually proved useful in characterising the properties of drugs. In this respect, electrochemical techniques for studying ion partitioning can be viewed as complimentary to traditional techniques, rather than as a replacement for them, and combining the two has resulted in a large expansion in the information which can be gleaned from ion partition studies. 


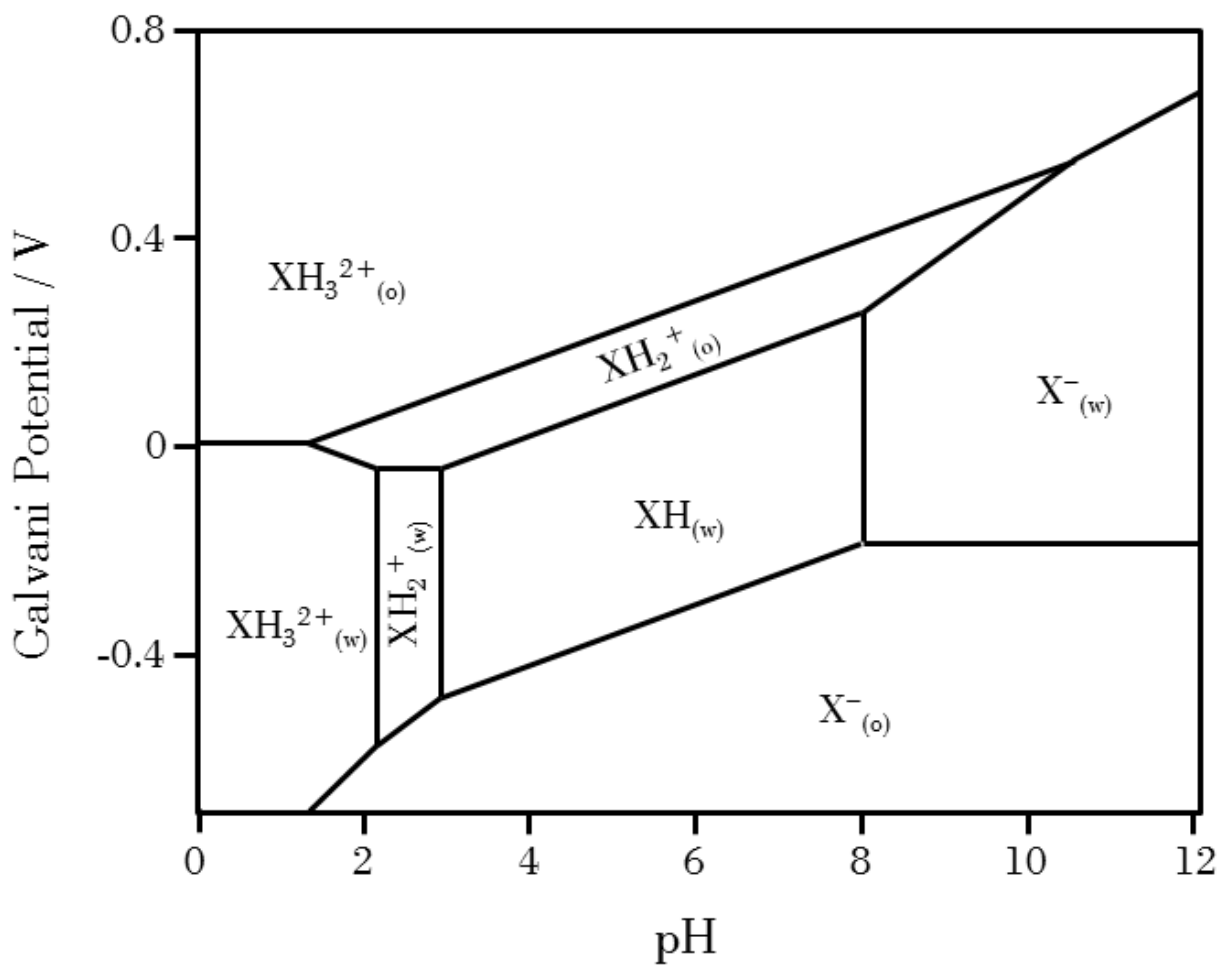

Figure 3. Theoretical ionic partition diagram of cetirizine $(X)$ based on analysis in [19].

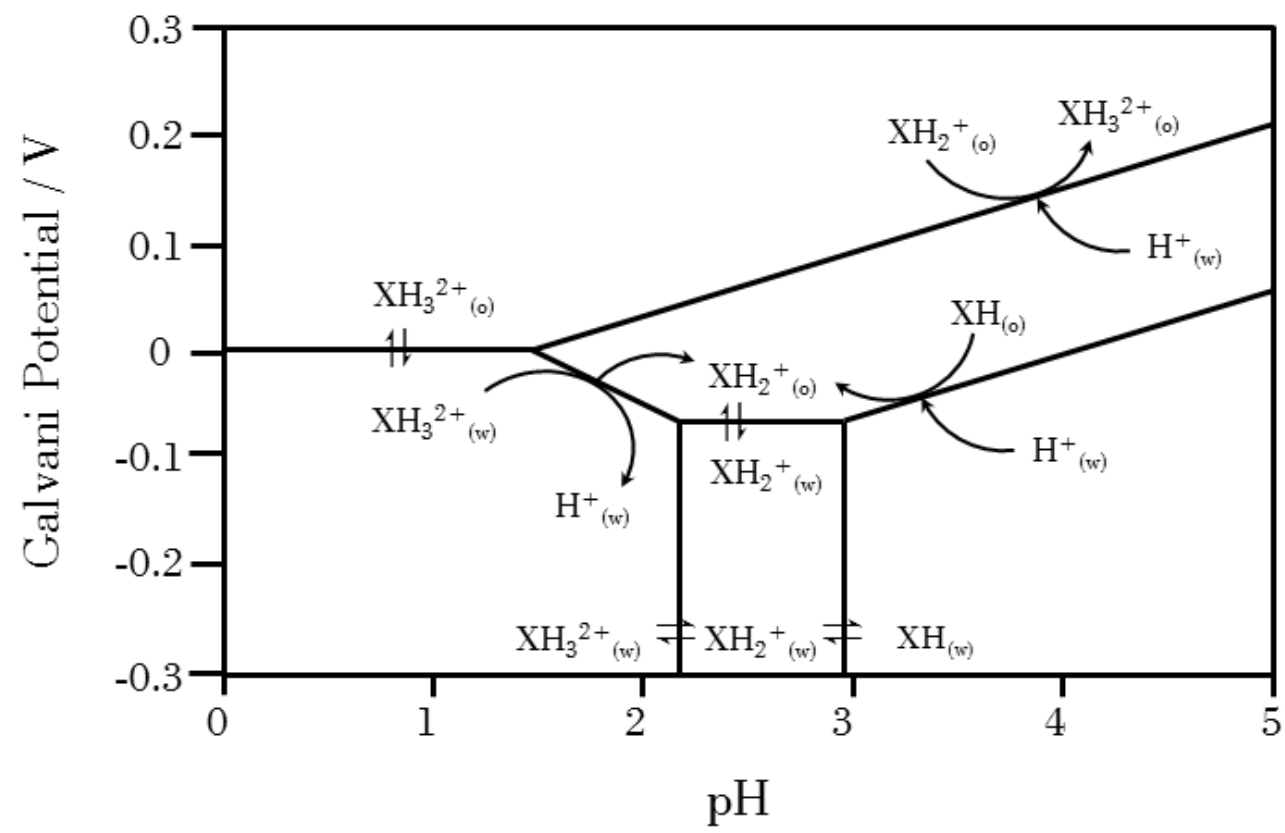

Figure 4. Detailed ionic partition diagram of cetirizine $(X)$, showing the mechanisms of phase transfer (based on analysis in [19]).

\section{Determination of diffusion coefficients}

In addition to the determination of partition coefficients, electrochemistry at the ITIES is ideally suited for measurement of the diffusion coefficients of ions. As charge transfer processes across the ITIES are diffusion controlled, the same equations that govern diffusion-controlled charge transfer at the solid electrode|solution interface also apply to the ITIES, namely the Randles-Ševčík equation (3) and the Cottrell equation (4) [13-15]. 


$$
\begin{gathered}
I_{\mathrm{p}}=0.4463\left(\frac{z_{\mathrm{i}} F}{R T}\right)^{1 / 2} z_{\mathrm{i}} F A D_{\mathrm{i}}^{1 / 2} c_{\mathrm{i}} v^{1 / 2} \\
I=\frac{z_{\mathrm{i}} F A c_{\mathrm{i}} \sqrt{D_{\mathrm{i}}}}{\sqrt{\pi t}}
\end{gathered}
$$

where $I_{\mathrm{p}}$ is the peak current observed from the voltammogram, $z_{\mathrm{i}}$ is the charge of the ion $\mathrm{i}, F=96485 \mathrm{C}$ $\mathrm{mol}^{-1}$ is the Faraday constant, $R=8.31 \mathrm{~J} \mathrm{~mol}^{-1} \mathrm{~K}^{-1}$ is the universal gas constant, $T$ is the temperature, $A$ is the ITIES area, $D_{\mathrm{i}}$ is the diffusion coefficient of the ion $\mathrm{i}, c_{\mathrm{i}}$ is the concentration of the ion $\mathrm{i}, v$ is the scan rate, $l$ is the time-dependent current and $t$ is time.

When determining the diffusion coefficient from the Randles- Ševčík equation, cyclic voltammetry is used and the scan rate is varied. A plot of $I_{p}$ against $v^{1 / 2}$ then yields a linear plot, the slope of which can be used to calculate the diffusion coefficient of ion i. Chronoamperometry is the technique employed when using the Cottrell equation for diffusion coefficient determination. The Galvani potential is stepped from a value at which the ion of interest does not transfer across the ITIES, to one at which it does. The second potential is then held for a predetermined amount of time and the diffusion controlled current decay is recorded. A plot of $I$ against $t^{-1 / 2}$ will then yield a linear plot, from which the diffusion coefficient of ion i can be calculated.

Although diffusion coefficients are important drug transport parameters, the number of studies determining their values using electrochemistry at the ITIES are negligible compared to partition coefficient studies. Kontturi and Murtomaki [10] did report diffusion coefficients in their original study, but stated that the determined values may not have been accurate due to a difference in drug concentration between the bulk and the liquid|liquid interface. Since then, the diffusion coefficients of drugs have largely been ignored in work at the ITIES. No explanation was given as to why such a concentration difference may have existed, but it proved to be of critical importance in a later study by Velický et al. [30].

In the work of Velický et al. [30], warfarin and propranolol were studied at $\mathrm{pH} 7.4$, at which both drugs are almost entirely ionised ( $0.26 \%$ and $0.74 \%$ neutral form of warfarin and propranolol, respectively). Using the ITIES setup described earlier, diffusion coefficients, which were 14- and 46-fold lower than predicted by molar mass [31], were determined for warfarin and propranolol, respectively. It was observed that the magnitude of the current produced by ion-transfer, which is used to calculate the diffusion coefficient of the ion, gradually decreased over time after initial set-up of the experiment and without an applied potential. This was explained, and confirmed by shake-flask experiments, by a partitioning of the neutral fraction of the drug across the interface. The concentration of the neutral fraction is constantly replenished through the aqueous dissociation equilibrium (illustrated for warfarin),

$$
\mathrm{W}_{(\mathrm{aq})}^{-}+\mathrm{H}_{(\mathrm{aq})}^{+} \leftrightarrow \mathrm{HW}_{(\mathrm{aq})}
$$

where $\mathrm{W}=$ warfarin. Provided that the concentration of protons in the aqueous phase is sufficient, the concentration of the ionic form of the drug at the interface gradually depletes, resulting in lowered iontransfer current and thus a lower calculated diffusion coefficient than would be expected for the bulk concentration of drug. 
Stirring of the aqueous phase was devised as a solution to this problem, therefore minimizing the effect of diffusive mass transport and maintaining a constant concentration of the ionic form of the drug at the interface. Stirring was made possible by separation of the two liquid phases using a polyvinylidene fluoride (PVDF) membrane. A diagram of the experimental setup used in this study is shown in Fig. 5. Diffusion coefficients of warfarin and propranolol determined via this method were on the order of magnitude expected from their molar masses [31]. This setup offered a solution to a problem almost conspicuous in its absence from the literature, given the expected ease with which diffusion coefficients could be calculated in tandem with partition coefficients at the ITIES.

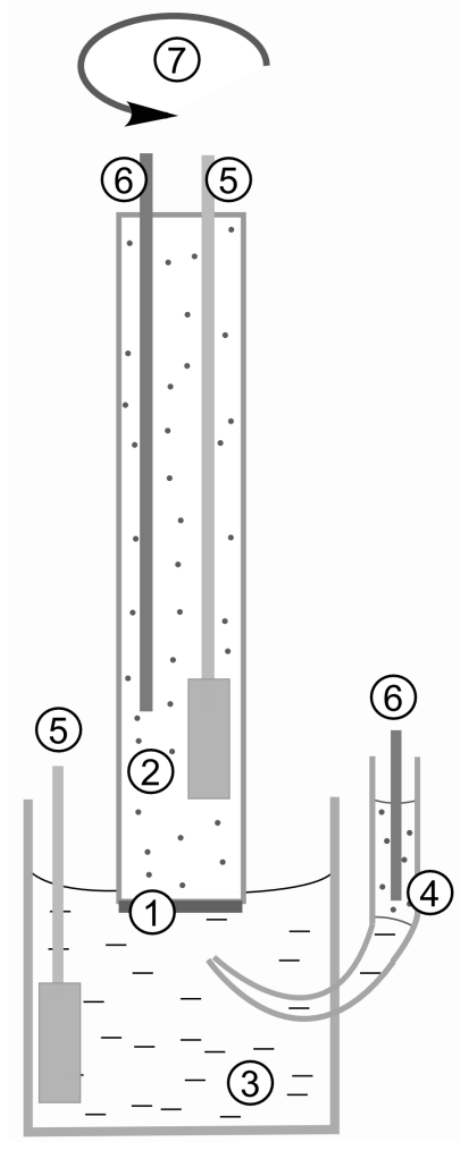

Figure 5. The schematic of the stirred ITIES setup used in [30], in which the two immiscible phases are separated by a rotating membrane. 1) PVDF membrane, 2) glass tube (aqueous phase), 3) glass cell (organic phase - DCE), 4) reference phase (aqueous), 5) counter electrodes (Pt), 6) reference electrodes ( $\mathrm{Ag} / \mathrm{AgCl})$, 7) rotation control.

\section{Voltammetry in membrane permeability studies}

Traditional bio-relevant membrane mimics such as black lipid membranes (BLM) and supported lipid membranes are invaluable substrates for studies of drug-membrane interaction and functions of biological membranes [32-34]. However, they are of a fragile nature, which inevitably limits their use. Supported liquid membranes (SLM) on the other hand, which typically contain a lipid solution of an organic solvent immobilised on a polymer membrane, are very stable and have intrinsic durability and are therefore suitable for use in the industrial setting and even large scale applications. SLMs were successfully applied in transfer studies of drug molecules, namely in prediction of drug absorption in humans using the artificial membrane permeability method Parallel Artificial Membrane Permeation Assay (PAMPA) [35-37], cellmonolayer permeability assays such as human epithelial colorectal adenocarcinoma (Caco-2)[38-40] or 
Madin-Darby canine kidney (MDCK) [41], drug lipophilicity determination $[37,42,43$ ] and extraction/removal of drugs $[44,45]$.

Polarisation of SLMs has attracted significant interest in the last few decades [46]. Thompson et al. have reported one of the first electrochemical characterisations of drug transfer (amphotericin B and valinomycin) across various polymer filter membranes soaked in organic solvent and lipids and proposed the formation of micro-BLMs within the membrane [47]. The most common electrochemical methods employed are cyclic voltammetry and chronoamperometry, with applications ranging from drug partitioning studies and drug extraction to concentration of analytes in order to increase analytical sensitivity. Fig. 6 shows a typical cyclic voltammogram of the transfer of ions obtained in a SLM system. Solvents traditionally used in the pharmaceutical industry, due to their good bio-mimetic properties, are not usually suitable for quantitative electrochemical experiments in SLM, due to their low polarity ( $n$ octanol, as mentioned in Section 2) or miscibility with water, problematic in thin (10-100 $\mu \mathrm{m})$ membranes (DCE). 1,2-dichlorobenzene (ODCB), nitrobenzene (NB), nitrophenyl octyl ether (NPOE) or 1,9-decadiene have proven to be good alternative solvents [48].

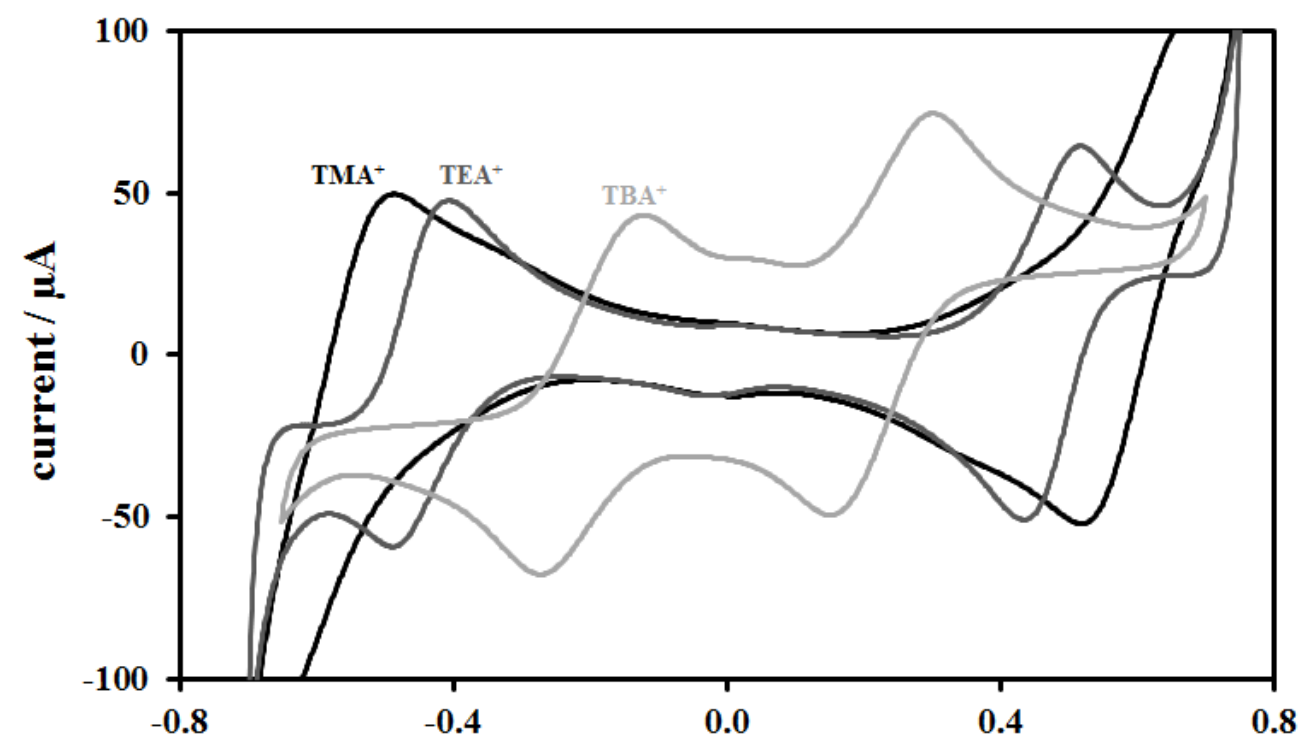

Galvani potential / V

Figure 6. Cyclic voltammograms of transfer of tetraalkylammonium ions across NPOE-based membrane at scan rates of $40 \mathrm{mV} \mathrm{s}^{-1}$. The ions are present on both sides of the membrane hence the double-peak transfer is observed (adapted from [48].

Electrochemistry in SLM systems is usually performed using a four-electrode setup, similar to that for a two-phase ITIES system described in Section 2 (Fig.1). A pair of reference and counter electrodes placed in each aqueous phase, which are separated by the immiscible organic membrane, as shown in Fig. 7.

Liquid/liquid extraction of drugs seems to be one of the most promising applications of SLMs in the pharmaceutical context. Pedersen-Bjergaard et al. have introduced a method of electro-kinetic migration of drugs across an NPOE-based membrane. Initially, a very large voltage of $300 \mathrm{~V}$ DC was applied and chemical tuning of the membrane composition used to extract both polar and non-polar drug molecules [49]. Gradually, the group improved the method by decreasing the voltage to values as low as $1 \mathrm{~V} \mathrm{DC} \mathrm{[50]} \mathrm{and}$ developing an on-chip device for online UV-vis and HPLC detection of electrically extracted drugs [51]. While this method is suitable for quantitative extraction of molecules across the membrane and promising 
for quick analysis of complex samples, such as urine or blood [52-54], the relatively high applied voltage and absence of supporting electrolyte in the membrane present a problem for theoretical explanation of the ionised drug transfer.

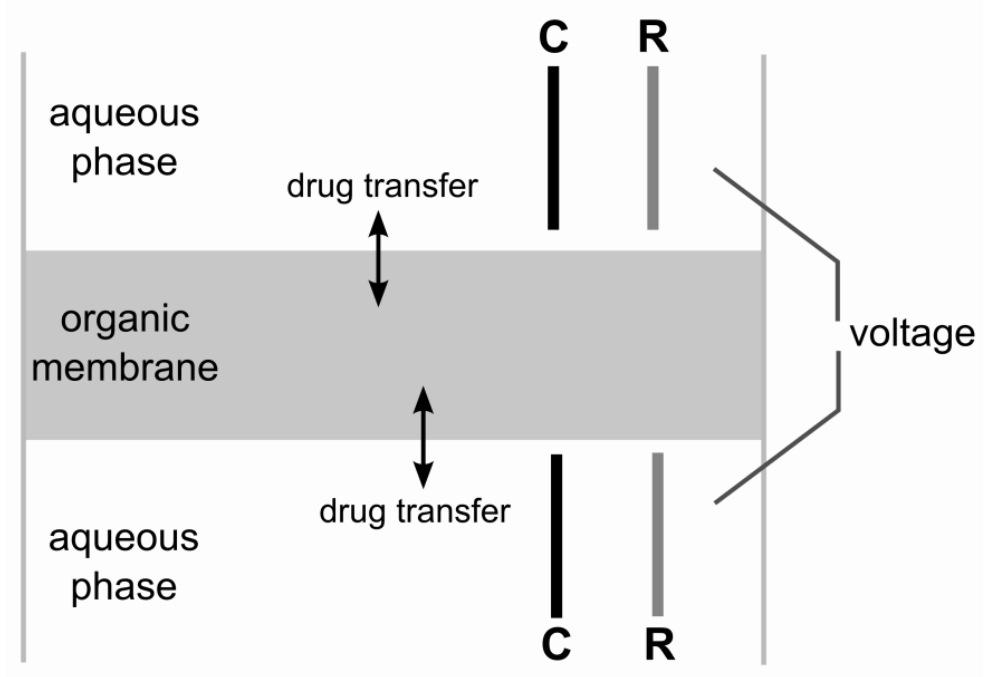

Figure 7. Schematic of a typical supported liquid membrane system. Voltage (or potential difference) across the liquid membrane is applied via a pair of reference and counter electrodes (denoted $R$ and $C$, respectively).

The fundamental understanding of electrochemically induced transfer across SLMs stems from electrochemistry at ITIES, as discussed in previous sections, and the connection between the two inspired researchers to extend the know-how to systems with two liquid|liquid interfaces, where a small voltage (usually below $500 \mathrm{mV} \mathrm{DC}$ ) is applied. Samec et al. studied transfer of protonated local anesthetics across an NPOE-based SLM using voltammetry and correlated their pharmacological potency data $[55,56]$ with the voltage required to transfer the drugs across the membrane, as shown in Fig. 8 [57]. Ulmeanu et al. used cyclic voltammetry and $\mathrm{pH}$ profiling to determine the partition coefficient of drugs using a commercial 96well micro-filter plate system, which by nature requires only a small amount of the organic solvent and studied drug [58]. Other reports made significant contribution to the polarised membrane research, albeit not directly studying pharmaceutically relevant molecules [59-63]. Significant efforts have also been spent on development of an electrochemical theory to describe the observed current-potential dependence, both for systems with and without membrane electrolytes [64-67]. Furthermore, a number of research activities have focused on use of SLMs as ion-selective electrodes applied to drug molecules [68].

The permeability of lipophilic drugs can be limited by transport across the aqueous phase boundary layer. It has been shown that controlled hydrodynamics or stirring of the membrane improved the predictive power of in vitro permeability assays, thus mimicking the diffusion/convection conditions in a human intestine $[69,70]$. The traditional view of the widely accepted $\mathrm{pH}$-partition hypothesis has also been challenged using a hydrodynamic approach to permeability [71]. It is interesting to note that several research groups independently pursued studies on the electrochemical response of SLM systems under hydrodynamic control. Manzanares et al. contributed an excellent research paper on determination of ion transfer kinetics in a rotated diffusion cell, i.e. with controlled rotation of the membrane phases, as well as analytical description of this system [72]. This work was followed by Murtomäki et al., who reported the effect of SLM composition on transfer of tetraalkylammonium and tacrine cations in the same system [73]. Most recently, permeation of an organic dye, crystal violet, was studied via UV-vis spectrophotometry and 
electrochemistry, with the transfer mechanism via naked ions and ion pairs across the membrane elucidated [74].

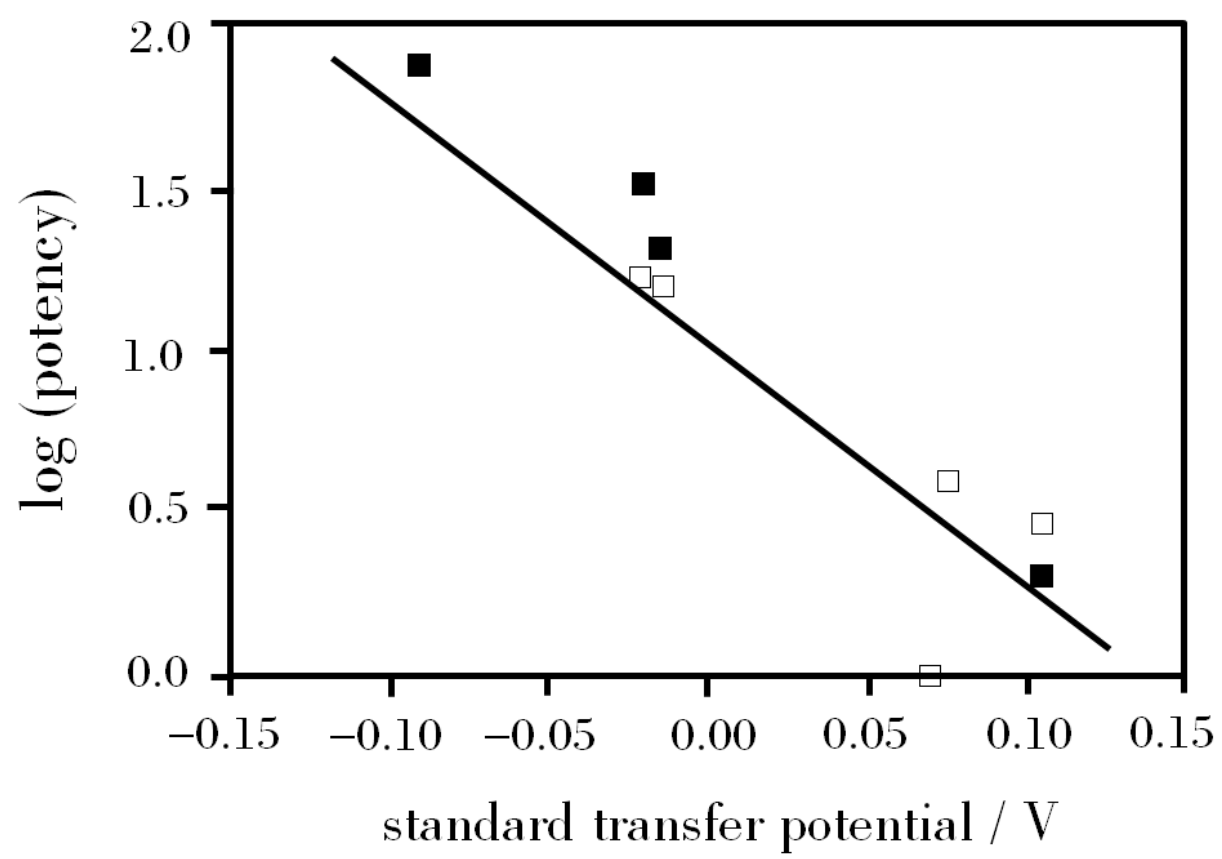

Figure 8. Logarithm of the drug potency relative to procaine vs. the standard ion transfer potentials of the protonated anesthetics: ( $\square$ ) potency taken from ref. [54], ( $\square$ ) expressed as the inverse ratio of the inhibition constants $I C_{50}$ [55].

There is yet to be a report to be published, in which all the above elements, i.e. membrane permeability, electrochemistry and hydrodynamic control, have been combined to study drug molecules and applied in a pharmaceutical setting. The strength of interdisciplinary collaboration has been proven in many research fields and so this opportunity, which could provide in-depth insight to drug-membrane interaction and significant improvement in prediction of drug in vivo behaviour, will hopefully not be overlooked.

\section{Conclusions}

In this mini review, we have shown the usefulness of ion-transfer voltammetry in studies of the distribution of ionisable drugs at the interface between two immiscible electrolyte solutions and related systems. Successful examples, particularly the determination of partition coefficients, diffusion coefficients and membrane permeability have been highlighted. It has been noted that the aforementioned voltammetric techniques coupled with controlled hydrodynamics or stirring of the membrane improve the predictive power of in vitro permeability assays, thus mimicking the diffusion/convection conditions in a human intestine. This novel approach offers a new way to study the passive transport of pharmaceutical compounds across biological membranes, which may facilitate the development of screening methods in pharmaceutical research.

\section{Acknowledgements:}

$M V$ and ANJR thank the EPSRC for funding. 


\section{References}

[1] J. Comer and K. Y. Tam, in Pharmacokinetic Optimization in Drug Research: Biological, Physicochemical and Computational Strategies (B. Testa, H. Van De Waterbeemd, G. Folkers, and R. Guy, eds.), Verlag Helvetica Chimica Acta, Zürich, 2001, p. 275.

[2] C. A. Lipinski, F. Lombardo, B. W. Dominy, and P. J. Feeney, Advanced Drug Delivery Reviews 46 (2001) 3-26.

[3] D. A. Smith and H. van de Waterbeemd, Current Opinion in Chemical Biology 3 (1999) 373-378.

[4] B. Testa, P. A. Carrupt, P. Gaillard, F. Billois, and P. Weber, Pharmaceutical Research 13 (1996) 335-343.

[5] J. C. Dearden and G. M. Bresnen, Quantitative Structure-Activity Relationships 7 (1988) 133-144.

[6] S. D. Krämer, J. C. Gautier, and P. Saudemon, Pharmaceutical Research 15 (1998) 1310-1313.

[7] A. Leo, C. Hansch, and D. Elkins, Chemical Reviews 71 (1971) 525-616.

[8] A. Mälkiä, L. Murtomäki, A. Urtti, and K. Kontturi, European Journal of Pharmaceutical Sciences 23 (2004) 13-47.

[9] K. Takacs-Novak and A. Avdeef, Journal of Pharmaceutical and Biomedical Analysis 14 (1996) 14051413.

[10] K. Kontturi and L. Murtomaki, Journal of Pharmaceutical Sciences 81 (1992) 970-975.

[11] F. Reymond, P. A. Carrupt, B. Testa, and H. H. Girault, Chemistry - A European Journal 5 (1999) 39-47.

[12] Z. Samec, J. Langmaier, A. Trojánek, E. Samcová, and J. Málek, Analytical Sciences 14 (1998) 35-41.

[13] J. Koryta, Electrochim Acta 24 (1979) 293-300.

[14] F. Reymond, D. Fermin, H. J. Lee, and H. H. Girault, Electrochimica Acta 45 (2000) 2647-2662.

[15] Z. Samec, Pure and Applied Chemistry 76 (2004) 2147-2180.

[16] P. Vanýsek, Electrochimica Acta 40 (1995) 2841-2847.

[17] P. Vanýsek and L. B. Ramírez, Journal of the Chilean Chemical Society 53 (2008) 1455-1463.

[18] G. Bouchard, A. Pagliara, P. A. Carrupt, B. Testa, V. Gobry, and H. H. Girault, Pharmaceutical Research 19 (2002) 1150-1159.

[19] G. Bouchard, A. Pagliara, G. P. Van Balen, P. A. Carrupt, B. Testa, V. Gobry, H. H. Girault, G. Caron, G. Ermondi, and R. Fruttero, Helvetica Chimica Acta 84 (2001) 375-387.

[20] H. Nagatani, S. Suzuki, D. J. Fermín, H. H. Girault, and K. Nakatani, Analytical and Bioanalytical Chemistry 386 (2006) 633-638.

[21] G. Bouchard, P. A. Carrupt, B. Testa, V. Gobry, and H. H. Girault, Chemistry - A European Journal 8 (2002) 3478-3484.

[22] S. Fantini, J. Clohessy, K. Gorgy, F. Fusalba, C. Johans, K. Kontturi, and V. J. Cunnane, European Journal of Pharmaceutical Sciences 18 (2003) 251-257.

[23] G. Bouchard, P. A. Carrupt, B. Testa, V. Gobry, and H. H. Girault, Pharmaceutical Research 18 (2001) 702-708.

[24] P. Jing, M. Zhang, H. Hu, X. Xu, Z. Liang, B. Li, L. Shen, S. Xie, C. M. Pereira, and Y. Shao, Angewandte Chemie - International Edition 45 (2006) 6861-6864.

[25] R. Gulaboski, V. Mirčeski, and F. Scholz, Electrochemistry Communications 4 (2002) 277-283.

[26] G. Bouchard, A. Galland, P. A. Carrupt, R. Gulaboski, V. Mirceski, F. Scholz, and H. H. Girault, Physical Chemistry Chemical Physics 5 (2003) 3748-3751.

[27] F. Quentel, V. Mirčeski, and M. L'Her, Journal of Solid State Electrochemistry 12 (2008) 31-39.

[28] F. Reymond, G. Steyaert, P. A. Carrupt, B. Testa, and H. Girault, Journal of the American Chemical Society 118 (1996) 11951-11957.

[29] F. Reymond, V. Chopineaux-Courtois, G. Steyaert, G. Bouchard, P. A. Carrupt, B. Testa, and H. H. Girault, Journal of Electroanalytical Chemistry 462 (1999) 235-250.

[30] M. Velický, K. Y. Tam, and R. A. W. Dryfe, Journal of Electroanalytical Chemistry 683 (2012) 94-102.

[31] A. Avdeef, Expert opinion on drug metabolism \& toxicology. 1 (2005) 325-342. 
[32] A. Mälkiä, PhD Thesis, Helsinki University of Technology (2004) p.84.

[33] R. P. Richter, J. L. K. Him, and A. Brisson, Materials Today 6 (2003) 32-37.

[34] G. Van Meer, D. R. Voelker, and G. W. Feigenson, Nature Reviews Molecular Cell Biology 9 (2008) 112124.

[35] M. Bermejo, A. Avdeef, A. Ruiz, R. Nalda, J. A. Ruell, O. Tsinman, I. Gonzalez, C. Fernandez, G. Sanchez, T. M. Garrigues, and V. Merino, European Journal of Pharmaceutical Sciences 21 (2004) 429-441.

[36] K. Sugano, H. Hamada, M. Machida, H. Ushio, K. Saitoh, and K. Terada, International Journal of Pharmaceutics 228 (2001) 181-188.

[37] F. Wohnsland and B. Faller, Journal of Medicinal Chemistry 44 (2001) 923-930.

[38] P. Artursson and J. Karlsson, Biochemical and Biophysical Research Communications 175 (1991) 880885.

[39] A. Avdeef and K. Y. Tam, Journal of Medicinal Chemistry 53 (2010) 3566-3584.

[40] V. Fade, Journal of Pharmaceutical Sciences 87 (1998) 1604-1607.

[41] J. D. Irvine, L. Takahashi, K. Lockhart, J. Cheong, J. W. Tolan, H. E. Selick, and J. R. Grove, Journal of Pharmaceutical Sciences 88 (1999) 28-33.

[42] B. Faller, H. P. Grimm, F. Loeuillet-Ritzler, S. Arnold, and X. Briand, Journal of Medicinal Chemistry 48 (2005) 2571-2576.

[43] M. H. Abraham, H. S. Chadha, R. A. E. Leitao, R. C. Mitchell, W. J. Lambert, R. Kaliszan, A. Nasal, and P. Haber, Journal of Chromatography A 766 (1997) 35-47.

[44] Z. Lazarova, B. Syska, and K. Schügerl, Journal of Membrane Science 202 (2002) 151-164.

[45] Y. Yamini, C. T. Reimann, A. Vatanara, and J. A. Jönsson, Journal of Chromatography A 1124 (2006) $57-$ 67.

[46] K. Kontturi, L. Murtomäki, and J. A. Manzanares, Ionic Transport Processes: in Electrochemistry and Membrane Science, Oxford, 2008.

[47] M. Thompson, R. B. Lennox, and R. A. McClelland, Analytical Chemistry 54 (1982) 76-81.

[48] M. Velický, K. Y. Tam, and R. A. W. Dryfe, Analytical Chemistry 86 (2014) 435-442.

[49] A. Gjelstad, K. E. Rasmussen, and S. Pedersen-Bjergaard, Journal of Chromatography A 1124 (2006) 2934.

[50] I. J. Ø. Kjelsen, A. Gjelstad, K. E. Rasmussen, and S. Pedersen-Bjergaard, Journal of Chromatography A 1180 (2008) 1-9.

[51] N. J. Petersen, S. T. Foss, H. Jensen, S. H. Hansen, C. Skonberg, D. Snakenborg, J. P. Kutter, and S. Pedersen-Bjergaard, Analytical Chemistry 83 (2011) 44-51.

[52] M. Eskandari, Y. Yamini, L. Fotouhi, and S. Seidi, Journal of Pharmaceutical and Biomedical Analysis 54 (2011) 1173-1179.

[53] S. Nojavan and A. R. Fakhari, Journal of Separation Science 33 (2010) 3231-3238.

[54] K. F. Seip, J. Stigsson, A. Gjelstad, M. Balchen, and S. Pedersen-Bjergaard, Journal of Separation Science 34 (2011) 3410-3417.

[55] L. M. Hondeghem and R. D. Miller, in Basic and Clinical Pharmacology (B. G. Katzung, ed.), Prentice-Hall Int. Inc., New York, 1992, p. 363.

[56] E. T. McNeal, G. A. Lewandowski, J. W. Daly, and C. R. Creveling, Journal of Medicinal Chemistry 28 (1985) 381-388.

[57] Z. Samec, A. Trojánek, J. Langmaier, E. Samcová, and J. Málek, Electroanalysis 12 (2000) 901-904.

[58] S. M. Ulmeanu, H. Jensen, G. Bouchard, P. A. Carrupt, and H. H. Girault, Pharmaceutical Research 20 (2003) 1317-1322.

[59] M. H. Barker, L. Murtomäki, and K. Kontturi, Journal of Electroanalytical Chemistry 497 (2001) 61-68.

[60] J. Langmaier, K. Stejskalová, and Z. Samec, Journal of Electroanalytical Chemistry 496 (2001) 143-147. 
[61] S. M. Ulmeanu, H. Jensen, Z. Samec, G. Bouchard, P. A. Carrupt, and H. H. Girault, Journal of Electroanalytical Chemistry 530 (2002) 10-15.

[62] J. Langmaier and Z. Samec, Electrochemistry Communications 9 (2007) 2633-2638.

[63] O. Shirai, S. Kihara, Y. Yoshida, and M. Matsui, Journal of Electroanalytical Chemistry 389 (1995) 61-70.

[64] T. Kakiuchi, Electrochimica Acta 44 (1998) 171-179.

[65] A. Molina, J. A. Ortuño, C. Serna, E. Torralba, and J. Gonzalez, Electroanalysis 22 (2009) 1634-1642.

[66] A. Molina, C. Serna, J. Gonzalez, J. A. Ortuño, and E. Torralba, Physical Chemistry Chemical Physics 11 (2009) 1159-1166.

[67] Z. Samec, A. Trojánek, J. Langmaier, and E. Samcová, Journal of Electroanalytical Chemistry 481 (2000) 1-6.

[68] J. Guo and S. Amemiya, Analytical Chemistry 78 (2006) 6893-6902.

[69] A. Avdeef, P. E. Nielsen, and O. Tsinman, European Journal of Pharmaceutical Sciences 22 (2004) 365374.

[70] M. Velický, K. Y. Tam, and R. A. W. Dryfe, European Journal of Pharmaceutical Sciences 44 (2011) 299309.

[71] M. Velický, D. F. Bradley, K. Y. Tam, and R. A. W. Dryfe, Pharmaceutical Research 27 (2010) 1644-1658.

[72] J. A. Manzanares, R. Lahtinen, B. Quinn, K. Kontturi, and D. J. Schiffrin, Electrochimica Acta 44 (1998) 59-71.

[73] L. Murtomäki, M. H. Barker, J. A. Manzanares, and K. Kontturi, Journal of Electroanalytical Chemistry 560 (2003) 95-103.

[74] M. Velický, K. Y. Tam, and R. A. W. Dryfe, Analytical Chemistry 84 (2012) 2541-2547.

(C)2014 by the authors; licensee IAPC, Zagreb, Croatia. This article is an open-access article distributed under the terms and conditions of the Creative Commons Attribution license (http://creativecommons.org/licenses/by/3.0/)(cc)) EY 